BULLETIN OF THE

AMERICAN MATHEMATICAL SOCIETY

Volume 81, Number 1, January 1975

\title{
COMPACT ANR'S HAVE FINITE TYPE ${ }^{1}$
}

\author{
BY JAMES E. WEST
}

Communicated October 2, 1974

In 1950, J. H. C. Whitehead [15] proved that compact metric ANR's have the homotopy types of (countable) cell complexes and asked whether they have the homotopy types of finite-dimensional cell complexes. In 1954 in his talk at the International Congress of Mathematicians in Amsterdam [2], Borsuk asked whether they have the homotopy types of finite complexes. The simply-connected case was answered in 1957 by de Lyra [9], and in 1965, Wall [14] produced an applicable obstruction theory for finiteness. That year, Mather settled the case of products of $S^{\mathbf{1}}$ [10], and a year later, Gersten's product formula [5] for Wall's obstruction settled the case of products with polyhedra having zero Euler characteristic. Then Siebenmann proved it for a large class of (finite-dimensional) manifolds [13] and with Kirby extended this to all (finite-dimensional, compact) manifolds [12]. Finally, in 1973 Chapman [3] added Hilbert cube manifolds and locally triangulable spaces.

In this notice an affirmative solution of these questions is sketched, using the notions of cell-like mappings and Hilbert cube manifolds together with a recent result of $R$. Miller [11], which is a cell-like analog of Mather's theorem and provides the basic existence theorem for cell-like mappings used here.

A cell-like $(C E)$ mapping is a proper surjection such that each point-inverse has the shape of a point. Cell-like mappings between locally compact, separable metric ANR's are homotopy equivalences [6], [7], [8]. The strategy of this argument is to construct, for any compact ANR $A$ a $C E$ mapping $f$ : $M^{Q} \rightarrow A$ from some Hilbert cube manifold $M^{Q}$. Because $M^{Q}$ is homeomorphic, by Chapman's Triangulation Theorem, [3], to $K \times Q$ for some finite complex $K(Q$ is the Hilbert cube), $A$ must have the homotopy type of

AMS (MOS) subject classifications (1970). Primary 54C55, 55D15, 57A20, 57A60.

Key words and phrases. Compact ANR, homotopy type, cell-like mapping, Hilbert cube manifold, Hilbert cube manifold factor, mapping cylinder.

1 Partially supported by NSF Grants GP33031 and GP33031X, and completed during the Georgia Topology Conference at the University of Georgia, 1974, where partial results comprised the author's talk. 
$K$. To obtain this $C E$ map from Miller's result that (essentially) $A \times S^{\mathbf{1}}$ and the cone $C(A)$ of $A$ are $C E$-images of Hilbert cube manifolds (finite complexes in the finite-dimensional case), the global property is reduced to a local one about Hilbert cube manifold factors which allows the restriction of attention to the base of the cone $C(A)$. The first step is a mapping cylinder theorem.

THEOREM 1. Let $f: M^{Q} \rightarrow A$ be a $C E$ mapping of a Hilbert cube manifold to a compact $A N R$ A. Then there is a homeomorphism from $M^{Q}$ to the mapping cylinder $M_{f}$ of $f$ which is homotopic to the natural inclusion $i: M^{Q} \rightarrow M_{f}$ of $M^{Q}$ into $M_{f}$.

This provides a link between $C E$ mappings and Hilbert cube manifolds. A subset $X$ of an ANR $Y$ is said to have Property $Z$ in $Y$ if it is closed and if for all open sets $U$ of $Y$ the inclusion $U \backslash X \rightarrow U$ is a homotopy equivalence. If $Y$ is a Hilbert cube manifold this is equivalent to the property that $X$ lies in a collared submanifold. In the mapping cylinder $M_{f}$ of Theorem 1 , the base (the natural copy of $A$ ) has Property $\mathrm{Z}$, a result obtained from an observation [4] of Doug Curtis-that the base $A$ has Property $\mathrm{Z}$ in $M_{f}$ if and only if $f$ is a. "fine homotopy equivalence", i.e. for every open cover $U$ of $A$, there is a homotopy inverse $g$ of $f$ such that the homotopies to the identity of $f g$ and $g f$ may be limited by $U$ and $f^{-1}(U)$-and the result of Haver [6] that $f$ is a fine homotopy equivalence if and only if $C E$ (see also Lacher [7], [8]). With a little Hilbert cube manifold theory, this and Theorem 1 yield the local characterization of ANR $C E$ images of compact Hilbert cube manifolds.

THEOREM 2. For a compact metric ANR $A$, the following are equivalent:

(1) $A$ is the CE image of a Hilbert cube manifold;

(2) the union of any two Hilbert cube manifolds along a copy of $A$ which has Property $\mathrm{Z}$ in each is a Hilbert cube manifold factor; and

(3) there exist two Hilbert cube manifolds whose union along a common copy of $A$ is a Hilbert cube manifold factor.

(The space $X$ is here defined to be a Hilbert cube manifold factor if $X \times Q$ is a Hilbert cube manifold.) Coupling Theorem 2 with Miller's theorem and regarding $C(A)$ as $A \times[0,1] / A \times\{0\}$, it is easy to demonstrate, using the Homogeneity Theorem of Anderson and Chapman [1], that 
THEOREM 3. Every compact metric $A N R$ is the $C E$ image of a Hilbert cube manifold.

This has, as indicated above, the following corollary:

COROLlaRY. Every compact metric ANR is homotopy-equivalent to a compact polyhedron.

\section{REFERENCES}

1. R. D. Anderson and T. A. Chapman, Extending homeomorphisms to Hilbert cube manifolds, Pacific J. Math. 38 (1971), 281-293. MR 47 \#7749.

2. K. Borsuk, Sur l'élimination de phenomènes paradoxaux en topologie générale, Proc. Internat. Congr. Math., Vol. I, Amsterdam, 1954, pp. 197-208.

3. T. A. Chapman, Compact Hilbert cube manifolds and the invariance of Whitehead torsion, Bull. Amer. Math. Soc. 79 (1973), 52-56.

4. D. W. Curtis, Talk to Louisiana State University problem session in infinite-dimensional topology, October 1973.

5. S. M. Gersten, A product formula for Wall's obstruction, Amer. J. Math. 88 (1966), 337-346. MR 33 \#6623.

6. W. Haver, Mappings between ANR's that are fine homotopy equivalences (preprint).

7. R. C. Lacher, Cell-like mappings. I, Pacific J. Math. 30 (1969), 717-731.

8. - Cell-like mappings. II, Pacific J. Math. 35 (1970), 649-660.

9. C. B. de Lyra, On spaces of the same homotopy type as polyhedra, Bol. Soc. Math. São Paulo 12 (1957), 43-62.

10. M. Mather, Counting homotopy types of manifolds, Topology 4 (1965), 93-94.

11. R. Miller, Mapping cylinder neighborhoods of some ANR's, Bull Amer. Math. Soc., (this issue)

12. R. C. Kirby and L. C. Siebenmann, On the triangulation of manifolds and the Hauptvermutung, Bull. Amer. Math. Soc. 75 (1969), 742-749. MR 39 \#3500.

13. L. C. Siebenmann, On the homotopy type of compact topological manifolds, Bull. Amer. Math. Soc. 74 (1968), 738-742. MR 37 \#3567.

14. C. T. C. Wall, Finiteness conditions for CW-complexes, Ann. of Math. (2) 81 (1965), 56-69. MR 30 \#1515.

15. J. H. C. Whitehead, $A$ certain exact sequence, Ann. of Math. (2) 52 (1950), 51-110. MR 12, 43 .

DEPARTMENT OF MATHEMATICS, CORNELL UNIVERSITY, ITHACA, NEW YORK 14850 